\title{
AUS DER PRAXIS
}

\section{Integrations-Tarifvertrag Auto 5000}

Kaum ein tarifpolitisches, beschäftigungspolitisches und arbeitsorganisatorisches Projekt fand so viel öffentliche Aufmerksamkeit wie das VW-Projekt $5000 \times 5000$ (Auto 5000). Das gilt für den Tarifkonflikt zu Beginn des Projektes 2001 genauso wie für die Erkenntnisse der mehrjährigen Projektlaufzeit und letztlich auch für die erfolgreiche Beendigung des Projektes und die Integration der Auto-5000-Belegschaft in die Volkswagen AG zu Beginn des Jahres 2009.

\section{PROJEKTSTART VON $5000 \times 5000$}

Der Vorstand von Volkswagen unterbreitete 2001 der IG Metall und dem Betriebsrat folgendes Konzept: Von der ursprünglich beschlossenen Fertigung des Minivan Touran in Ost-Europa könne Abstand genommen werden, wenn die Fertigung auf dem Werksgelände in Wolfsburg in einer separaten Einheit mit einem gesonderten Tarifvertrag außerhalb des Haustarifvertrages der Volkswagen AG erfolgen würde. Langfristig könnten 5.000 Arbeitslose für ein Einheitsentgelt von seinerzeit DM 5.000 beschäftigt werden. Zunächst würden 3.500 Arbeitslose in Wolfsburg eingestellt und durch eine mehrmonatige Qualifizierung in die Lage versetzt, den Touran zu produzieren. Darüber hinaus wollte Volkswagen neue Formen der Arbeitsorganisation und Qualifizierung erproben.

Das Modell enthielt eine Reihe interessanter und innovativer Aspekte. Andererseits stellte es den offensichtlichen Versuch dar, tarifliche Mindeststandards zu unterlaufen. Die Aussicht, 3.500 arbeitslosen Menschen die Perspektive von unbefristeten Normalarbeitsverhältnissen zu ermöglichen, führte aber dazu, dass die IG Metall mit Volkswagen Verhandlungen aufnahm und ein Gegenmodell vorstellte.

Die überaus konfliktreichen Tarifverhandlungen wurden von beiden Seiten

\begin{tabular}{|c|c|}
\hline \multicolumn{2}{|c|}{ Übersicht 1: Meilensteine des Projekts Auto 5000} \\
\hline November 1999 & $\begin{array}{l}\text { Erste Vorstellungen über die Planung des Projektes } 5000 \text { X } 5000 \text { durch den } \\
\text { damaligen Arbeitsdirektor der Volkswagen AG }\end{array}$ \\
\hline Februar 2001 & Volkswagen stellt sein Konzept vor \\
\hline März 2001 & Die IG Metall stellt ihr Konzept vor \\
\hline Mai 2001 & Beginn der Tarifverhandlungen \\
\hline Juni 2001 & Vorläufiges Scheitern der Tarifverhandlungen \\
\hline August 2001 & Abschluss eines Projekt-Tarifvertrages \\
\hline März 2002 & Beginn der Rekrutierung von Arbeitslosen \\
\hline November 2002 & Produktionsstart des Touran \\
\hline September 2005 & Tarifvertrag zur Produktion des Tiguan \\
\hline Juli 2006 & $\begin{array}{l}\text { Erste eigenständige Tarifrunde der Belegschaft der Auto 5000; } \\
\text { Abschluss eines Manteltarifvertrages und Entgelttarifvertrages }\end{array}$ \\
\hline Mai 2007 & $\begin{array}{l}\text { Tarifrunde: Entgelttarifvertrag und Zwischenschritte zur Heranführung } \\
\text { an den Volkswagen-Tarifvertrag }\end{array}$ \\
\hline August 2007 & Produktionsstart des Tiguan \\
\hline November 2008 & Abschluss des Integrationstarifvertrages \\
\hline Januar 2009 & $\begin{array}{l}\text { Integration von ca. } 3.500 \text { Beschäftigten der Auto } 5000 \text { in die Volkswagen AG, } \\
\text { zunächst noch mit befristeten Übergangsregelungen bezüglich Entgelt und } \\
\text { Schichtmodellen }\end{array}$ \\
\hline Januar 2011 & $\begin{array}{l}\text { Vollständige Anwendung der Tarifverträge der Volkswagen AG für die } \\
\text { ehemaligen Beschäftigten der Auto } 5000\end{array}$ \\
\hline
\end{tabular}

Mitte 2001 für gescheitert erklärt und konnten erst in einem zweiten Anlauf abgeschlossen werden.

Das IG Metall-Tarifsystem für das Volkswagen Projekt $5000 \times 5000$ ist an anderer Stelle ausführlich dokumentiert ${ }^{1}$ und soll hier nur stichwortartig in Erinnerung gerufen werden:

- 3.500 Arbeitslose werden in die neu gegründete Auto $5000 \mathrm{GmbH}$ in Wolfsburg eingestellt, sechs Monate lang qualifiziert und danach in ein unbefristetes Arbeitsverhältnis übernommen.

- Die Arbeitszeit beträgt 35 Stunden pro Woche mit erweiterten Flexibilisierungsmöglichkeiten. Die Produktion des Touran erfolgt in einem klassischen Drei-SchichtSystem unter Einbeziehung der Samstagsfrühschicht und teilweise auch der Samstagsspätschicht.

- Für die Beschäftigten in der Produktion wird kein übliches System von Entgeltgruppen vereinbart. Stattdessen wird ein Einheitsentgelt von 5.000 DM (2.556€) gezahlt, mit denen alle Schichtzuschläge abgegolten sind.

- Parallel zur Produktion erfolgt eine prozessorientierte Qualifizierung von insgesamt drei Stunden pro Woche, die zur Hälfte vom Unternehmen bezahlt und zur Hälfte von den Beschäftigten eingebracht werden. Im Tarifvertrag werden Prinzipien

\footnotetext{
1 Meine, H./Schwitzer, H. (2001): Das IG MetallSystem für das Volkswagen-Projekt $5000 \times 5000$, in: WSI-Mitteilungen 9, S. 580-582. Zu den wichtigsten Meilensteinen des Projekts vgl. Übersicht 1.
}

Hartmut Meine, Bezirksleiter des IG Metall Bezirkes Niedersachsen und Sachsen-Anhalt, Verhandlungsführer für die Tarifverhandlungen der IG Metall bei der Volkswagen AG. e-mail: hartmut.meine@igmetall.de Thilo Reusch, Bezirkssekretär des IG Metall Bezirkes Niedersachsen und Sachsen-Anhalt, zuständig für die Tarifpolitik bei der Volkswagen AG.

e-mail: thilo.reusch@igmetall.de 
einer ganzheitlichen Arbeitsorganisation verankert, beispielsweise abwechslungsreiche und ganzheitliche Arbeitsinhalte, Teamarbeit, lernförderliche Arbeitsorganisation und flache Hierarchien.

- Für die Leistungs- und Personalbemessung wird vereinbart, dass Management und Betriebsrat Arbeitspensum und Personalbemessung für die jeweiligen Bereiche vereinbaren. Kriterien sind betriebswirtschaftliche Vorgaben, arbeitswissenschaftliche Erkenntnisse sowie die Einhaltung der regelmäßigen Arbeitszeit und der Pausen.

- In einem gesonderten Mitbestimmungsvertrag sind besondere Rechte des Aufsichtsrates, erweiterte Mitbestimmungsrechte des Betriebsrates und ein Benachteiligungsverbot für gewerkschaftliche Funktionäre abgesichert.

In einem aufwendigen, systematischen Auswahlverfahren wurden 3.500 arbeitslose Menschen ausgewählt. Der zweite Teil des Projektes, der weiteren 1.500 Menschen Arbeit bringen sollte, wurde nicht realisiert. Die neu eingestellten Arbeitslosen wurden in einem mehrmonatigen Qualifizierungsprogramm befähigt, im November 2002 mit der Produktion des neuen Minivan Touran zu beginnen. Der erfolgreiche Produktionsstart widerlegte die Aussagen der Zweifler. Sie hatten öffentlich behauptet, dass es trotz Qualifizierungsmaßnahmen nicht gelingen könne, wettbewerbsfähige Fahrzeuge ausschließlich mit ehemaligen Arbeitslosen zu fertigen.

\section{AUTO 5000: EIN NEUES PRODUKTIONSKONZEPT}

Da bei der Arbeitsorganisation und dem Produktionskonzept Neuland betreten wurde, gab es von Beginn an eine wissenschaftliche Begleitforschung und Evaluation des Projekts durch das Soziologische Forschungsinstitut an der Universität Göttingen (SOFI). Die Finanzierung des Forschungsprojekts wurde gemeinsam von der Hans-Böckler-Stiftung, der IG Metall und der Volkswagen AG getragen; die Ergebnisse sind an anderer Stelle ausführlich dokumentiert. ${ }^{2}$ Hier sollen nur wenige Erkenntnisse in Erinnerung gerufen werden:

(1) Die Qualifizierung der vormals arbeitslosen Beschäftigten zu Beginn des Projektes und die dabei eingesetzten Methoden sind überaus positiv zu bewerten und haben sich bewährt. Es ist gelungen, inner- halb von sechs Monaten die Beschäftigten zu qualifizieren, in Großserienfertigung Fahrzeuge in der erforderlichen Qualität und Stückzahl herzustellen. Während des laufenden Betriebes fand eine Weiterqualifizierung parallel zum Produktionsprozess statt. Dazu wurden neben den Montagelinien sogenannte Lernfabriken installiert, sodass es möglich war, in unmittelbarer Nähe der Produktion fertigungsnahe Qualifizierungsprozesse durchzuführen. Nachdem ein hoher Qualifizierungsstand der Belegschaft erreicht war, nahm der erforderliche Umfang der Weiterqualifizierung allerdings ab und beschränkte sich überwiegend auf die Qualifizierung bei Neuanläufen.

(2) Die Prinzipien einer ganzheitlichen Arbeitsorganisation wurden nach den Erkenntnissen der Begleitforschung bei Auto 5000 wesentlich stärker und konsequenter umgesetzt als in anderen Bereichen der Automobilindustrie. Dies gilt insbesondere für ganzheitliche Produktionsaufgaben, die volle Rotation in einer Gruppe sowie die Übertragung von Prozessoptimierungsaufgaben an die Beschäftigten. Die Forscher stellten ein hohes Niveau bei der Gruppenselbstorganisation ebenso fest wie intensive Kooperationsmöglichkeiten in Zusammenarbeit mit gewählten integrierten Gruppensprechern. Die erste Führungsebene (Betriebsingenieure) erhielt ein völlig neues Aufgabenprofil, das die klassische Meisterfunktion wesentlich erweiterte.

\section{TARIFRUNDEN 2004 BIS 2007}

In der Zeit von 2004 bis 2007 fanden mehrere entscheidende Ergänzungen des Tarifsystems statt. Zunächst wurde 2005 vereinbart, das Produktspektrum der Auto 5000 um ein weiteres Fahrzeug zu erweitern. Neben dem Minivan Touran fertigten die Auto-5000-Beschäftigten ab August 2007 den kleinen Geländewagen Tiguan. Vor dem Hintergrund einer schwierigen wirtschaftlichen Situation bei Volkswagen wurde zudem vereinbart, dass zwei Ausbildungsjahrgänge nicht von der Volkswagen AG, sondern von der Auto 5000 in ein unbefristetes Arbeitsverhältnis übernommen wurden.

Im Jahre 2006 kam es zur ersten konfliktreichen Tarifrunde der bisher in Tarifauseinandersetzungen unerfahrenen Auto5000-Belegschaft. Nach mehreren Verhandlungen und mehreren Warnstreiks unter Beteiligung fast aller Beschäftigten konnte der Projekttarifvertrag abgelöst und durch einen Manteltarifvertrag und Entgelttarifvertrag für die Auto 5000 ersetzt werden. Durch die vereinbarten Entgelterhöhungen von $3 \%$ erhöhten sich das Monatsentgelt und die zusätzlichen Jahresboni. ${ }^{3}$ In der folgenden Tarifrunde des Jahres 2007 konnte das Tarifsystem der Auto 5000 schrittweise an den Volkswagen-Tarifvertrag herangeführt werden. So wurden beispielsweise vermögenswirksame Leistungen und eine betriebliche Altersversorgung eingeführt.

\section{INTEGRATION DER AUTO 5000 IN DIE VOLKSWAGEN AG}

Bereits zu Beginn des Projektes stellte sich die Frage, ob es dauerhaft möglich ist, auf einem Werksgelände zwei Belegschaften mit zwei unterschiedlichen Tarifsystemen zu beschäftigen. Die erwarteten Konflikte blieben aber zunächst aus. Mit der Etablierung des Auto-5000-Projektes forderten die Beschäftigten aber immer deutlicher die Gleichbehandlung mit der VolkswagenBelegschaft. Der Wunsch, von Auto 5000 nach Volkswagen zu wechseln, wurde zum Diskussionsthema in der Belegschaft. Nachdem es gelungen war, in der Tarifverhandlung 2007 eine weitere Angleichung der tariflichen Bedingungen an das VolkswagenTarifsystem durchzusetzen, verstärkte sich diese Diskussion abermals. Durch die Neujustierung des VW-Haustarifvertrages 2006 wurde der Abstand zum Tarifvertrag der Auto 5000 geringer, sodass sich die Chancen für eine Angleichung verbesserten. Hinzu kam, dass sich mit der Entscheidung, den Tiguan bei der Auto 5000 zu fertigen, die Rahmenbedingungen ganz erheblich veränderten: Während die Fertigung des Touran räumlich klar gegenüber der Volkswagen-Fertigung abgegrenzt war, musste für die Produktion des Tiguan ein neuer Rohbau mehrere hundert Meter von

\footnotetext{
2 Schumann, M./Kuhlmann, M./Sanders, F./Sperling, H. J. (2005): Anti-Tayloristisches Fabrikmodell - Auto 5000 bei Volkswagen, in: WSI-Mitteilungen 1, S. 3-10; Schumann, M./Kuhlmann, M./ Sanders, F./Sperling, H. J. (2006): Vom Risikozum Vorzeigeprojekt: Auto 5000 bei Volkswagen, in: WSI-Mitteilungen 6, S. 299-306; Schumann, M./Kuhlmann, M./Sanders, F./Sperling, H. J.: (Hrsg.) (2006): Auto 5000: Ein neues Produktionskonzept - die deutsche Antwort auf den Toyota-Weg?, Hamburg.

3 Vgl. Meine, H./Schwitzer, H. (2006): Neuland erfolgreich bearbeitet, in: Schumann, M. et al., vgl. Fn. 2.
} 
den Auto-5000-Hallen aufgebaut werden. Zudem mussten für die Montage des Tiguan Kapazitäten im Montagebereich von Volkswagen erweitert werden, sodass im Wechsel Tiguan und Golf gefertigt werden konnten. Zudem kam es zu einem vermehrten Personalwechsel zwischen der Auto 5000 und der Volkswagen AG, der sich tarif- und arbeitsrechtlich kompliziert gestaltete. Diese Konstellation förderte die Aufnahme von Tarifverhandlungen der IG Metall mit Volkswagen, an deren Ende im November 2008 ein Tarifvertrag zur Integration der Auto-5000-Beschäftigten in die Volkswagen AG stand.

\section{ECKPUNKTE DES INTEGRATIONS- TARIFVERTRAGES}

Allen 3.500 Auto-5000-Beschäftigten wurde Ende 2008 ein Arbeitsvertrag bei der Volkswagen AG angeboten. Erwartungsgemäß nahmen das Angebot alle Beschäftigten an. Damit gilt für die Auto-5000-Beschäftigten seit dem 1. Januar 2009 der Haustarifvertrag der Volkswagen AG. Für den Zeitraum von zwei Jahren gelten aber noch Übergangsregelungen, die sich auf das Monatsentgelt und das derzeitige Schichtsystem beziehen. Die Anwendung des Haustarifvertrages führt aber bereits ab Januar 2009 zu einer deutlichen Erhöhung der monatlichen Entgelte. So werden etwa Schichtzuschläge und Pausenzeiten bezahlt. Während der zweijährigen Übergangszeit sind die Beschäftigten in die 20 Entgeltgruppen des Haustarifvertrages von Volkswagen einzugruppieren. Im Jahr 2011 wird die jeweilige Eingruppierung wirksam werden und zu differenzierten, nach Tätigkeit unterschiedlich hohen Entgelterhöhungen führen. Für Volkswagen ergeben sich mit der Integration der Auto 5000 Möglichkeiten eines flexibleren Personaleinsatzes am Standort Wolfsburg. Die bei der Auto 5000 implementierten Elemente einer ganzheitlichen Arbeitsorganisation haben Bestand. Im Rahmen des Tarifabschlusses von 2006 wurden für die Beschäftigten der Volkswagen AG Grundsätze einer innovativen Arbeitsorganisation vereinbart. Diese sind in der Betriebsvereinbarung „Volkswagen-Weg“ konkretisiert, wie beispielsweise die Prinzipien der Teamarbeit.

Es bleibt abzuwarten, inwieweit es gelingt, die Prinzipien einer ganzheitlichen Arbeitsorganisation flächendeckend bei Volkswagen zu realisieren.

\section{FAZIT}

Zum Abschluss des siebenjährigen Auto5000-Projektes ist ein positives Fazit zu zie- hen. Es ist gelungen, mit 3.500 ehemals arbeitslosen Menschen eine wettbewerbsfähige Serienproduktion am Standort in Wolfsburg zu tariflichen Bedingungen zu realisieren. In sieben Jahren haben die Beschäftigten bewiesen, dass Menschen, die in ihrer vorherigen Erwerbsbiografie nicht im Automobilbau beschäftigt waren, qualifiziert werden können, um hochwertige Produkte zu fertigen.

Mit der Integration von Auto 5000 werden im Stammwerk von Volkswagen in Wolfsburg pro Jahr insgesamt ca. 750.000 Fahrzeuge produziert. Damit ist das Wolfsburger Werk der zweitgrößte Automobilstandort der Welt, der sich durch zahlreiche Optimierungsprozesse einschließlich Auto 5000 in den letzten Jahren mehrfach selbst erneuerte. „Propheten“ im Management und der Wissenschaft, die dem Werk in Wolfsburg noch vor wenigen Jahren keine Zukunftschancen mehr gegeben hatten, sind durch die Praxis widerlegt. Es ist möglich, am Standort Deutschland im Rahmen einer 33- bzw. 35-Stunden-Woche und mit einem Entgelt-Niveau, das über dem Flächentarifvertrag liegt, wettbewerbsfähig Automobile zu produzieren. 